\title{
AN INDUCTIVE METHOD OF CALCULATION OF THE DEFLECTION OF THE TRUSS REGULAR TYPE
}

\author{
Mikhail Kirsanov \\ Moscow Power Engineering Institute (National Research University) \\ 14 Krasnokazarmennya str., Moscow, 111250, Russia
}

c216@ya.ru

\begin{abstract}
In this paper, the author proposes a regular option of statically determinate flat truss with a lattice of Shukhov's type working under beam scheme. By induction, using Maple computer algebra system, the exact formula for the mid-span deflection under the action of the uniform load is obtained. The deflection of the elastic structure is defined by the formula of Maxwell - Mohr. Forces in the rods are found using cut nodes method with the composition of the management of the matrix of equations in vector form. The analysis of the coefficients in the truss evaluation with a different number of panels revealed the recurrence equation satisfied by these coefficients, and then the determined operators of a specialized package genfunc give a solution of the resulting equation that determines the required general formula. The case of a uniform load on the top horizontal zone is considered. The author obtained a simple asymptotic evaluation of this solution, proving the cubic nature of the growth of deflection for a fixed span length and a given nodal load. The solution found for an even number of panels and rods of the same cross-section, has a polynomial form; the dependence is non-monotonic and detects extremum, which allows optimizing the design.
\end{abstract}

Keywords: truss, deflection, induction, exact solution, Maple.

\section{Introduction}

The number of diagrams of statically determinate trusses of the regular type, which used in mechanical engineering as elements of bearing systems, lifting mechanisms of lightweight truss manipulators is very limited. Based on them more complex (regular or irregular) statically indefinable constructions are created. Solutions for basic systems can be used as a part of the solution for the real system by the method of forces or just as a test, and debug for various kinds of numerical methods or software packages based on them. The problem of finding diagrams of statically determinate trusses is called "hunting", and this "hunting" is keep going (Hutchinson and Fleck, 2005). In this paper, author proposes a new scheme of the regular type, where the cell performs the regular Shukhov' truss. This type of truss doesn't allow the direct computation of forces in the rods (regardless of the current load). In this paper we consider regular truss (Figure 1). Denote $n$ is the number of panels (cell periodicity in the form of Shukhov's truss).

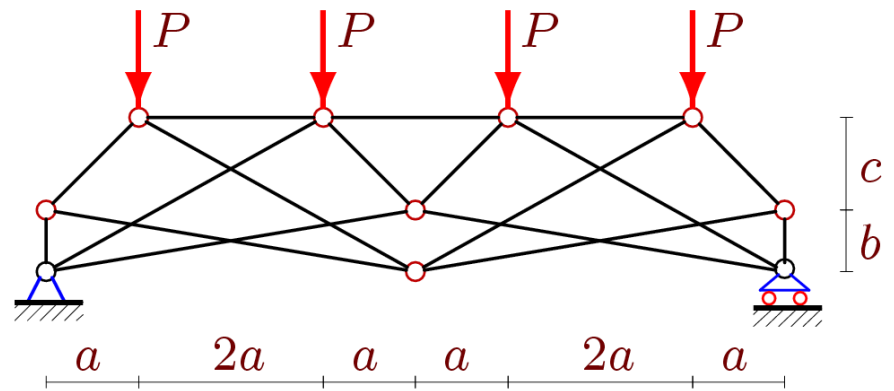

Figure 1. Truss, $k=1$

The method of cutting out of knots in the form in which it is usually applied (calculation from node to node with a consistent definition of the forces of the "chain") are not suitable. There are no such nodes which are attached to only two rods with unknown forces and with which to be- 
gin the calculation. The method of sections (Ritter's method) is also not suitable since the respective cross sections for $n>1$ does not exist. Only the calculation of the complete system of equilibrium equations of all components and definition forces across all terminals, including three rods, simulating the support is possible. This approach will be used below. Moreover, the aim is not just to obtain a formula for a specific truss, but to deduce the General formula for all trusses of this scheme, i.e. to obtain the solution for an arbitrary number of panels. The solution will be obtained by the methods of symbolic mathematics using the method of induction. Previously this method was used for deriving the formulas of deflection in a plane and spatial structures (Kirsanov, 2014, 2015a,b; Tinkov, 2015).

The geometry of the truss and the system of equations of equilibrium

We will create the system of equilibrium equations of all the nodes in the projections on the coordinate axes to compute the forces in the rods using cut nodes. The origin of coordinates we choose the left fixed support. Calculation program is compiled in the Maple language (Monagan et al., 2005). Assume that the truss contains an even number of panels: $n=2 k$. The number of the rod is $n_{s}=8 n+4$, including three support rods. The number of nods along with three nods mounted on the base $m=4 n+5$. We will set coordinates of nodes on which it will be possible to determine the directing cosines of the forces included in the equation of equilibrium:

$$
\begin{aligned}
& x_{i}=4 a(i-1), y_{i}=0, \\
& x_{i+n+1}=x_{i}, \quad y_{i+n+1}=b, i=1, \ldots, n+1, \\
& x_{i+2 n+2}=a+2 a(i-1), y_{i+2 n+2}=b+c, i=1, \ldots, 2 n .
\end{aligned}
$$

Coordinates of the supports:

$$
\begin{aligned}
& x_{m-2}=-2, y_{m-2}=0, \\
& x_{m-1}=x_{1}, y_{m-1}=-1, \\
& x_{m}=x_{n+1}, y_{m}=-1 .
\end{aligned}
$$

The order of connection of nodes and cores in the truss we will introduce by special vectors $\bar{V}_{i}, i=1, \ldots, n_{s}$. Here the first component is the number of the joint at the beginning of the corresponding rod, the second is the number of the hinge at the end. Of course, the choice of special direction vectors of the rods is not associated with the sign of force in them. We have the following vectors:

$$
\begin{aligned}
& \bar{V}_{i}=[i, 2(i+n+1)], \bar{V}_{i+n}=[i, i+n+2], \quad \bar{V}_{i+2 n}=[i+1, i+n+1], \\
& \bar{V}_{i+3 n}=[i+1,2(i+n)+1], \bar{V}_{i+4 n}=[i+n+1,2(i+n)+1], \\
& \bar{V}_{i+5 n}=[i+n+2,2(i+n+1)], \quad i=1, \ldots, n, \\
& \bar{V}_{i+6 n}=[i+2(n+1), i+2 n+3], i=1, \ldots, 2 n-1 .
\end{aligned}
$$

The following vectors present the vertical side rods of the truss:

$$
\bar{V}_{8 n}=[1, n+2], \bar{V}_{8 n+1}=[1+n, 2 n+2] .
$$

Three rods of the supports correspond to the following vectors:

$$
\bar{V}_{n_{s}-2}=[1, m-2], \quad \bar{V}_{n_{s}-1}=[1, m-1], \quad \bar{V}_{n_{s}}=[n+1, m] .
$$

The length of the rods and the projection of the vector representations of these rods need to calculate the direction cosines:

$l_{i}=\sqrt{l_{1, i}{ }^{2}+l_{2, i}{ }^{2}}, l_{1, i}=x_{V_{2, i}}-x_{V_{1, i}}, l_{2, i}=y_{V_{2, i}}-y_{V_{1, i}}, \quad i=1, \ldots, n_{s}$.

The first index in the number $V_{j, i}$ takes on the values 1 or 2 and corresponds to the number of components of the vector $V_{i}$; the second is a number of the rod. The matrix $\Phi$ guides of the cosines has the following elements

$\Phi_{k, i}=-l_{j, i} / l_{i}, k=2 V_{i, 2}-2+j, k \leq n_{s}, j=1,2, i=1, \ldots, n_{s}$,

$\Phi_{k, i}=l_{j, i} / l_{i}, \quad k=2 V_{i, 1}-2+j, \quad k \leq n_{s}, \quad j=1,2, i=1, \ldots, n_{s}$.

The forces can be determined as:

$$
\Phi \bar{S}=\bar{B},
$$

where $S=\left\{S_{1}, \ldots, S_{n_{s}}\right\}$ is the vector of forces in rods, $B=\left\{P_{x, 1}, P_{y, 1}, \ldots, P_{x, n_{s}}, P_{y, n_{s}}\right\}$ is the vector of the right parts (external loads applied to nodes). In order to calculate the deflection of the truss by the formula of Maxwell - Mohr: $\Delta=P \sum_{i=1}^{n_{s}-3} S_{i}^{(P)} S_{i}^{(1)} l_{i} /(E F)$, it is necessary to determine the force $S_{i}^{(1)}$ from the action of a single force applied at the middle of the lower zone (unit $k+1$, Figure 2). $S_{i}^{(P)}$ is the force in the rods from a given load and calculates the length of the rods $l_{i}$. The rigidity of the rods EF accepts the same.

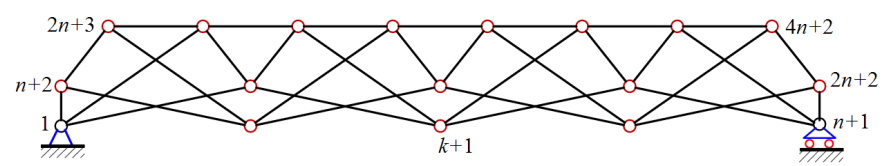

Figure 2. The numbering of the nodes in the truss, $n=2 k=4$

\section{Solution}

We consider the case of loading of the upper belt truss when $b=c$. In this case, the non-zero elements of the vector of the right part of the system of equilibrium equations of the nodes will have the following: $B_{2 i}=-P, i=2 n+3, \ldots, 4 n+2$. Solution (1) with such righthand side gives the vector of forces $\bar{S}^{(P)}$. The vector $\bar{S}^{(1)}$ of forces from a single power at node $k+1$ selected at $B_{2(k+1)}=1$ (the other elements are zero). The calculation is produced in the analytical form consistently for a truss with two panels, then four, three, etc. In every solution it is possible to identify specific components and their ratios. The deflection of the truss with $\mathrm{k}$ panels in half of the span takes the form

$$
E F \Delta_{k}=P\left(A_{k} a^{3}+B_{k} b^{3}+G_{k} g^{3}+D_{k} d^{3}+H_{k} h^{3}\right) /\left(25 b^{2}\right),
$$

where $d=\sqrt{16 a^{2}+b^{2}}, g=\sqrt{a^{2}+b^{2}}, h=\sqrt{9 a^{2}+b^{2}}$ is the length of the braces. 
The regularity in the sequence of coefficients can be found by the methods of Maple computer algebra system. With the command rgf_findrecur of package genfunc for next sequence $225,4400,21925,69600,169325, \ldots$ 17753600 of $A k$ of length 16 we obtain the recurrence equation as follows:

$$
A_{k}=2 A_{k-1}+2 A_{k-2}-6 A_{k-3}+6 A_{k-5}-2 A_{k-6}-2 A_{k-7}+A_{k-8}
$$

The operator rsolve gives the solution of this equation. The following general member of the sequence has the form:

$$
A_{k}=25\left(65 k^{4} / 6+\left(3(-1)^{k}+5\right) k^{2} / 12+(-1)^{k}-1\right) .
$$

In a similar way the next coefficients are obtained:

$$
\begin{aligned}
& B_{k}=(25 / 2) k\left(1-(-1)^{k}\right), \\
& G_{k}=(10 / 3) k^{4}+\left((-1)^{k}+23 / 3\right) k^{2}+3\left(1-(-1)^{k}\right), \\
& D_{k}=(10 / 3) k^{4}+\left((-1)^{k}+1 / 6\right) k^{2}-3\left(1-(-1)^{k}\right) / 4, \\
& H_{k}=(5 / 6) k^{4}+\left(3(-1)^{k}+53\right) k^{2} / 12-3\left(1-(-1)^{k}\right) .
\end{aligned}
$$

\section{Analysis}

Graph of (2) deflection $\Delta=\Delta E F / P_{\text {sum }}$ from the number of panels with constant span length $L=4 a n=40 \mathrm{~m}$ and a given total magnitude of the vertical load $P_{\text {sum }}=2 n P$ (Figure 3 , the dimensions in meters) shows that this dependence is non-monotonic for small heights of the truss. The kinks of the curves appear for small k and define "flashing" terms $(-1)^{k}$ in the coefficients. Of course, the initial part of the curve with $b=1 \mathrm{~m}$ has no practical value, because the corresponding panel length is too big for this altitude truss $a=40 / 8=5 \mathrm{~m}$, but the fact of crossing curves can be used in a practical sense in case of choosing the optimal size of a truss. The dependence of the deflection height (Figure 4) shows a clear minimum, which can also be used to optimize the structures. For a larger number of panels, extremum is stronger. It is interesting to perform the marginal analysis of the obtained formula. In the Maple system, using the limit operator (Delta/ $k^{\wedge} 3, k=$ infinity) we find that the increase of deflection is cubic $\lim _{k \rightarrow \infty} \tilde{\Delta} / k^{3}=2 b / 15$ and it is determined only by the height of the truss.

\section{Conclusions}

We proposed and calculated analytically statically determinate trusses in Maple regular scheme. Although the machines and mechanisms, in which the use of such schemes is assumed, undergo mainly dynamic loads, the static solution makes sense as a base, or estimated for more complex tasks, loads and types of supports. The solution has a polynomial form; the dependence is non-monotonic and detects extremum, which allows optimizing the design. The author provided the plots of deflection against a number of panels and height of the truss, showing the link between the deflection number of panels and height of the truss. Calculation and analysis are performed for a particular case when

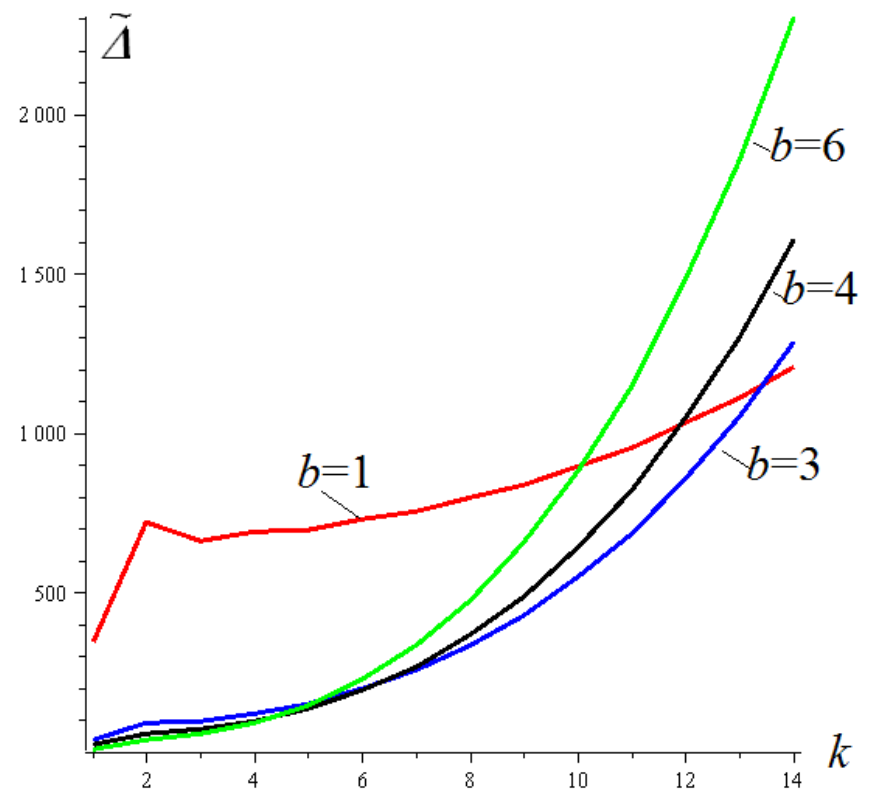

Figure 3. The dependence of the deflection on the number of panels

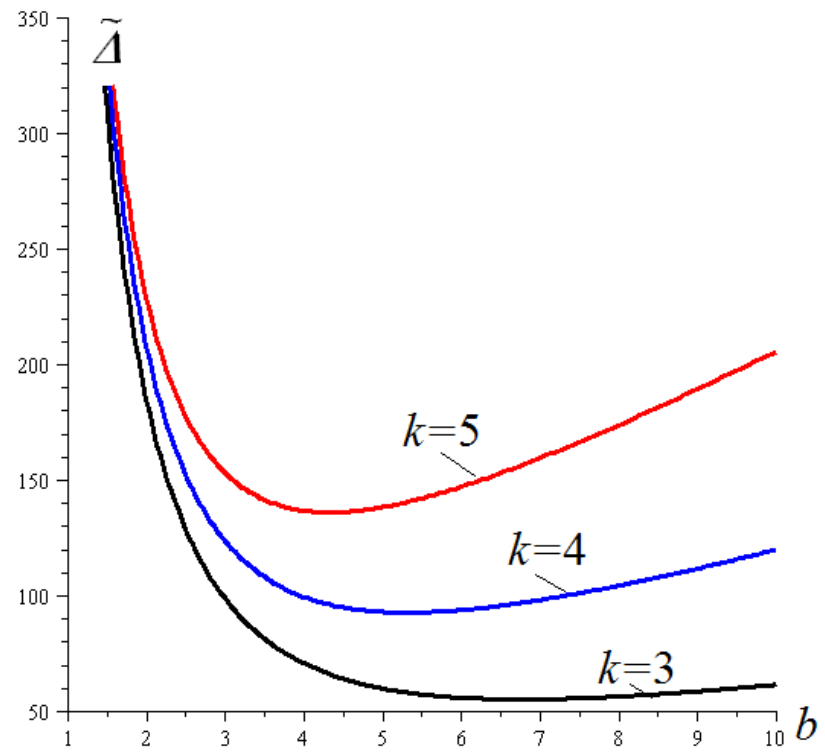

Figure 4. The dependence of deflection from the height

$b=c$. Based on the proposed algorithm, this solution can be easily generalized to any instance. However, the final formula, in this case, will be more complex. Marginal analysis revealed the cubic nature of the change of the deflection depending on the number of panels fixed on the length of the passage. Without any changes, the whole calculation can be performed in other symbolic mathematics packages. Similar design with precise formulas can be embedded in building structures to give them architectural expression and optimum rigidity. The analysis and optimization of beam structures was examined in a number of related researches (Farshi and Alinia-ziazi, 2010; Heyman, 2010; Kaveh and Talataharib, 2009). 


\section{References}

Farshi, B., Alinia-ziazi, A. (2010). Sizing optimization of truss structures by method of centers and force formulation. International Journal of Solids and Structures, 47, pp. 2508 - 2524. doi:10.1016/j.ijsolstr.2010.05.009

Heyman, J. (2010). Design of a simple steel truss. Proceedings of the Institution of Civil Engineers: Structures and Buildings, 163(1), pp. 53 - 56. doi: 10.1680/stbu.2009.163.1.53

Hutchinson, R.G., Fleck, N.A. (2005). Microarchitectured cellular solids - the hunt for statically determinate periodic trusses. ZAMM Z. Angew. Math. Mech., 85(9), pp.: 607 - 617. doi: 10.1002/zamm.200410208

Kaveh, A., Talataharib, S. (2009). Size optimization of space trusses using Big Bang-Big Crunch algorithm. Computers and Structures, 87, pp. 1129 - 1140. doi:10.1016/j.compstruc.2009.04.011

Kirsanov, M.N. (2014). Bending, torsion and the asymptotic analysis of the spatial core console [lzgib, kruchenie i asimptoticheskij analiz prostranstvennoj sterzhnevoj konsoli]. Magazine of Civil Engineering, 5(49), 37 - 43. doi: 10.5862/MCE.49.4 (in Russian).

Kirsanov, M.N. (2015a). Deflection analysis of rectangular spatial coverage truss [Analiz progiba fermy pryamougol'nogo prostranstvennogo pokrytiya]. Magazine of Civil Engineering, 1(53), pp. 32-38. doi: 10.5862/MCE.53.4 (in Russian).

Kirsanov, M.N. (2015b) Analysis of the deflection of a strut-type lattice girder truss [Analiz progiba reshetchatoj balochnoj fermy raspornogo tipa]. Magazine of Civil Engineering, 5(57), pp. 58 - 65. doi: 10.5862/MCE.57.5 (in Russian).

Monagan, M.B., Geddes, K.O., Heal, K.M., Labahn, G., Vorkoetter, S.M., McCarron, J., DeMarco, P. (2005). Maple 10 Advanced Programming Guide, Waterloo Maple Inc.

Tinkov, D.V. (2015). Comparative analysis of analytical solutions to the problem of truss structure deflection [Sravnitel'nyj analiz analiticheskih reshenij zadachi o progibe fermennyh konstrukcij], Magazine of Civil Engineering, 5(57), pp. 66 - 73. doi: 10.5862/ MCE.57.6 (in Russian). 